Théologiques

Théologiques

\title{
La mise en discours de l'humain chez saint Paul et ses interprétations anthropologiques en christianisme
}

Relecture de 1 Co $6,12-20$; 1Co 2,10-3,4 et 1Co 15,35-53

\section{Alain Gignac}

Volume 12, numéro 1-2, 2004

Le Soi dans tous ses états

URI : https://id.erudit.org/iderudit/011557ar

DOI : https://doi.org/10.7202/011557ar

Aller au sommaire du numéro

\section{Éditeur(s)}

Faculté de théologie et de sciences des religions, Université de Montréal

\section{ISSN}

1188-7109 (imprimé)

1492-1413 (numérique)

Découvrir la revue

Citer cet article

Gignac, A. (2004). La mise en discours de l'humain chez saint Paul et ses interprétations anthropologiques en christianisme : relecture de 1Co 6,12-20 ; 1Co 2,10-3,4 et 1Co 15,35-53. Théologiques, 12(1-2), 95-124.

https://doi.org/10.7202/011557ar

Tous droits réservés (C) Faculté de théologie et de sciences des religions, Université de Montréal, 2005
Ce document est protégé par la loi sur le droit d'auteur. L'utilisation des services d'Érudit (y compris la reproduction) est assujettie à sa politique d'utilisation que vous pouvez consulter en ligne.

https://apropos.erudit.org/fr/usagers/politique-dutilisation/ 


\title{
La mise en discours de l'humain chez saint Paul et ses interprétations anthropologiques en christianisme ${ }^{1}$
} Relecture de 1Co 6,12-20; 1Co 2,10-3,4 et 1Co 15,35-53

\author{
Alain GignaC \\ Faculté de théologie et \\ de sciences des religions \\ Université de Montréal
}

\begin{abstract}
[Scriptural teaching on the nature of the person] is one of the most vexing issues in the discussion, in part because our current questions were not the questions of the writers of the Scripture, but also because of a long story of projecting onto the texts interpretations and even translations that reflect later writers' concerns and assumptions. (Murphy 1999, 4)

Suis-je un corps? Ai-je un corps? Les deux. Sans le système symbolique du langage je ne pourrais pas dire «je». Mais sans le corps organique, je ne pourrais pas non plus dire «je». (Vergote 1979, 175)
\end{abstract}

Depuis Rudolph Bultmann (1969), c'est devenu un cliché d'affirmer qu'on lit toujours la Bible avec des présupposés (choix de méthodes et hypothèses de lecture), voire une précompréhension (intérêt pour la Bible), mais qu'il y a toujours un risque que cela ne soit pas mis au clair et devienne préjugé. On lit alors dans la Bible ce que l'on sait de toute manière y trouver. Au lieu d'être à l'écoute du texte, on lui impose un métadiscours qui lui est étranger. Au lieu de faire de l'acte de lecture une expérience imprévue, stimulante et dérangeante, on prend appui sur le texte pour conforter ses conceptions sur Dieu, sur l'humain.

1. La problématique et les conclusions de cet article ont été présentées au colloque «Le Soi dans tous ses états» (Montréal, 18-19 septembre 2003). 
La contribution de la Bible au débat sur le Soi peut s'échouer sur cet écueil. Par exemple, d'aucuns considéreront évident que la Bible propose une conception fonctionnelle de l'humain en termes dualistes, corps / âme (encore récemment, Cooper 2001). Cet article invite tous ceux qui participent au débat à remettre en question les évidences, afin de réviser une perception souvent simplificatrice de l'anthropologie chrétienne en général, et biblique en particulier.

\section{Préambule: un dialogue entre théologie et neurosciences?}

J'ai longtemps hésité, quant à savoir si je placerais ce qui suit en préambule ou en finale de ma présentation de l'anthropologie paulinienne, ou même s'il convenait de l'y incorporer. D'emblée, je prends donc le risque de poser l'impertinente question: le dialogue entre théologie et neurosciences peut-il être fécond? Est-il même possible? Autrement dit, en quoi un exposé biblique peut-il contribuer aux observations empiriques et à leurs modélisations, opérées par les neurosciences? Ou en quoi celles-ci viennent-elles interférer avec ma pratique de lecture des textes pauliniens?

Certains répondraient du tac au tac: "en rien », mettant presque un terme à la discussion, ou tout au moins établissant entre les deux domaines une séparation étanche ${ }^{2}$. D’autres, reprenant la distinction héritée de Wilhelm Dilthey entre expliquer et comprendre, distingueraient deux niveaux d'appréhension du réel (les sciences s'occupant du "comment?" et la religion du "pourquoi?»), avec le même résultat. Or cette distinction est de plus en plus remise en question, tant du côté de l'herméneutique biblique, qui conjugue expliquer et comprendre comme deux moments logiques en corrélation ${ }^{3}$, que du côté des sciences elles-mêmes,

2. À l'occasion du colloque "Le Soi dans tous ses états", alors que je soulevais ce genre de questions, Henri Atlan (qui contribue aussi à ce numéro) me répondait en substance: "Quant à la recherche en neuroscience, les religions ne sont d'aucune utilité, puisqu'elles n'opèrent pas sur le même plan; ce n'est que devant les limites [bioéthiques] des sciences qu'il convient d'écouter les religions. »

3. Ricoeur (1986) parle d'arc herméneutique: précompréhension / explication / compréhension, où l'explication doit impérativement prendre en compte les données des sciences mais s'avère insuffisante, car elle doit conduire à une appropriation où le lecteur se comprend lui-même devant le texte et en interaction avec lui. 
qui ne réduisent pas leur quête au seul aspect heuristique, mais redécouvrent pour ainsi dire une préoccupation pour le comprendre.

Ces deux réponses, insuffisantes, sont donc à renvoyer dos à dos, au profit d'une "mutuelle interaction créatrice», selon l'expression de Ted Peters (2002, xii). Dans un schéma reproduit en annexe à cet article (fig. 2), Robert John Russel (2002, 12), fondateur du Center for Theology and the Natural Sciences (Berkeley), décrit ainsi cette corrélation: d'une part la théologie doit intégrer les observations des sciences ou leurs théorisations dans les données qu'elle interprète, ou les incorporer à titre de postulats philosophiques, ou encore s'en inspirer sur les plans conceptuels ou esthétiques; d'autre part la science peut intégrer une doctrine religieuse à ses a priori philosophiques, se laisser stimuler par une question théologique ou encore guider dans le discernement des possibles théories par une conviction de foi (qui devient un critère parmi d'autres, suggestif mais non absolu) ${ }^{4}$.

Pour ma part, je suis convaincu que la théologie chrétienne, à cause même de sa définition classique de "foi en quête d'intelligence ", ne peut que se confronter aux questions, aux acquis (toujours temporaires), voire aux intuitions des sciences et des philosophies de chaque époque d'ailleurs la première partie de cet essai l'illustrera amplement: la lecture des textes bibliques est teintée par l'épistémologie, la vision du monde et le savoir encyclopédique du lecteur. Inversement, il me semble que les questions, les convictions «argumentées» et les intuitions concernant l'être humain, que porte la théologie, peuvent alimenter l'arrière-fond conceptuel où se construit la recherche expérimentale. Les théologies chrétiennes (plurielles dans le temps et l'espace, y compris dans le corpus biblique, on le verra aussi) ne sont pas liées à un cadre ou à une théorie scientifique en particulier; de même, si les neurosciences ont jusqu'ici emprunté une perspective empiriste, peut-être que la confrontation à une anthropologie différente - par exemple, chrétienne ou bouddhiste ${ }^{5}-$ permettrait l'émergence de questions nouvelles (ce que l'on cherche) et la modélisation nouvelle des données recueillies (ce que l'on trouve).

4. On trouvera un exemple d'une démarche scientifique en nursing qui se laisse interpeller par différents modèles d'anthropologie religieuse chez Cossette et Pepin (2001).

5. Voir la contribution de Victor Sōgen Hori dans ce numéro. 
Une fois admise la nécessité du dialogue, il faut aussitôt reconnaître le défi que représente l'apprivoisement du vis-à-vis, de sa démarche et de son langage. À des neuroscientifiques dont les investigations m'éblouissent - au double sens du vertige et de l'admiration —, on me demande de donner accès à l'anthropologie chrétienne par le biais bien particulier des textes pauliniens du Nouveau Testament. Par souci pédagogique, j'entends donc ici insister sur trois idées. Premièrement, il n'existe pas «une» anthropologie chrétienne et chaque génération croyante transite par le texte paulinien pour y construire son anthropologie. Deuxièmement, le vocabulaire paulinien n'est pas technique mais fluctue d'un texte à l'autre, d'où la nécessité d'une lecture «sur texte " - notre échantillon se limitant à trois paragraphes d'une même lettre (1Co 6,12-20; 1Co 2,10-3,4 ; 1Co 15,35-53). Troisièmement, les mots utilisés par Paul vie animée (âme), chair, corps et souffle (esprit) — sont autant d'aspects de l'être humain dans son rapport à Dieu, rapport dramatiquement modifié et qualifié par «l'événement » Jésus Christ, c'est-à-dire l'interprétation de la mort de Jésus comme résurrection et donc comme avènement d'un cosmos transformé et d'une humanité nouvelle.

Ultimement, il s'agit de montrer qu'il existe certainement des intuitions pauliniennes au sujet de l'humain; qu'elles sont mises en discours de manière à décrire non pas tant les composantes de l'humain que son rapport à Dieu; qu'elles ont pu être intégrées diversement à différentes "anthropologies théologiques"; que les textes où elles s'inscrivent peuvent donc servir de catalyseurs à notre propre réflexion anthropologique aujourd'hui. Paul n'est pas un maître à pensées (qui fournirait un "prêtà-penser»), mais un maître à penser.

\section{Anthropologies plurielles}

Le constat est presque simpliste dans sa limpidité, mais il faut le rappeler: il n'existe pas une seule anthropologie chrétienne, du fait de la pluralité des confessions (catholique, orthodoxe, protestante) mais aussi des diverses mouvances ou tendances qui traversent chacune d'elles. Bien plus, la norme identitaire qu'est la Bible est elle-même plurielle, dans ses genres littéraires, ses constructions théologiques, sa longue maturation rédactionnelle (un millénaire!) et ses ancrages culturels multiples (cananéen, égyptien, babylonien, grec). 
On oppose généralement les anthropologies juive (biblique) et grecque, comme deux sources en tension où aurait puisé le christianisme. Or, il faut nuancer cette idée reçue ${ }^{6}$. Dans la mosaïque des judaïsmes du I ${ }^{\text {er }}$ siècle, qui constitue le terreau de Nouveau Testament, il existe plusieurs anthropologies ${ }^{7}$. Par exemple, les pharisiens croient à la résurrection et les sadducéens la refusent. De même, du point de vue hellénistique, la conception de l'humain mise de l'avant par le stoïcisme diffère de celle des disciples de Platon et d'Aristote. Le judaïsme est lui-même extrêmement hellénisé, les textes bibliques tardifs en témoignent, particulièrement les textes pauliniens qui nous occupent ici. Paul de Tarse, pharisien, rodé à l'interprétation juive des Écritures, écrit en grec et véhicule malgré tout dans ses lettres l'enseignement éthique éclectique de son époque, fortement teinté de stoïcisme ${ }^{8}$. Citoyen romain, il est au carrefour des deux cultures, comme les Juifs de New York aujourd'hui parlent, pensent et vivent à la manière états-unienne sans perdre pour autant leur judaïté.

Il n'est pas surprenant qu'un survol rapide de l'histoire de la théologie soit marqué lui aussi du sceau du pluralisme, puisque chaque époque relit cette Bible pluriforme à partir de sa propre anthropologie? Autrement dit, de manière plus positive, et pour s'en tenir à Paul, les grands penseurs utilisent ses textes comme catalyseur pour articuler et illustrer leur propre conception de l'humain.

- Augustin (354-430) pouvait ainsi retrouver ses conceptions d'inspiration néo-platonicienne en 2Co 5,6-7: "Ainsi, toujours pleins de hardiesse, et sachant que rester là dans ce corps, c'est rester en exil loin du Seigneur, car nous cheminons dans la foi, non dans la claire vision...» L'âme immortelle (mais non éternelle) utilise comme véhicule temporaire le corps ${ }^{10}$. Toutefois, une autre lecture

6. Pour une conclusion similaire, voir Green 1999, 8-11.

7. Voir Cavallin 1974. En faisant l'inventaire de la littérature paratestamentaire juive, l'auteur montre que les représentations de l'après-mort sont le plus souvent juxtaposées dans une même œuvre sans souci de synthèse.

8. En 1Co 6,12-20, qui sera étudié sous un autre biais, on discerne trois critères éthiques stoïciens: la liberté du sage, la considération du bénéfice d'une action et l'importance de la maîtrise de soi (Hays 1997).

9. Pour une présentation différente, voir Murphy (1999, iv-x) et Vergote (1979). Ce dernier insiste sur l'influence de Platon, d'Aristote et de Descartes dans le débat).

10. Remarquons ici qu'Augustin, ancien manichéen, prend ses distances par rapport à la conception platonicienne du corps prison ou tombeau de l'âme (selon l'apho- 
du même texte est possible, mettant plutôt l'accent sur le désir d'être-avec Christ et d'être-ensemble, à partir des métaphores bibliques de la tente (au désert) et du vêtement (baptismal) (Gignac 2001, 299-305).

- Thomas d'Aquin (1224-1274), à partir de l'aristotélisme hylémorphique, définit l'âme comme la forme du corps (Blais 1990, 111119), avec une anthropologie plus unitive, bien que tripartite, dont il peut retrouver l'écho en 1Th 5,23: «Que votre lot tout entier

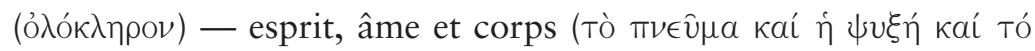
$\sigma \omega \mu \alpha)$ — soit gardé irréprochable. »

- Faisant un saut dans le temps, on constate que l'anthropologie holistique de Rudolph Bultmann (1884-1976) en termes d'êtredans-le-monde et d'être-de-relation, si influente en exégèse, subit elle-même l'influence de la philosophie existentiale de Heidegger (Bultmann, 1951 et 1955, 190-226) ${ }^{11}$.

- Enfin, des philosophes contemporains s'interrogent sur la condition politique de l'humain à partir de Paul (Gignac 2002). Alain Badiou (né en 1937) voit en Paul le modèle du sujet suscité par l'événement dont il témoigne, sur l'arrière fond d'une nouvelle métaphysique qui oppose être et événement (Badiou 1998), tandis que Giorgio Agamben (né en 1942), qui se pose en héritier de Walter Benjamin, voit en Paul le théoricien du sujet messianique qui reçoit un appel qui révoque toute vocation (Agamben 2000).

Comment expliquer que les mêmes textes puissent se prêter à des interprétations si contrastées? De fait, le corpus paulinien constitue une mise en discours non conceptuelle liée à des circonstances précises. Ce genre d'écriture tient plus de l'homélie que du traité systématique, ce qui fait sa force et sa faiblesse. Paul y répond à des questions ad hoc et ajuste son argumentation à son auditoire, en faisant flèche de tout bois. Il ne s'agit pas tant d'expliquer que de convaincre, afin d'induire un agir spécifique, porteur d'une éthique et d'une identité nouvelles enracinée dans l'expérience du Christ. Si Paul fait appel à des notions anthropologiques,

risme célèbre $\sigma \omega \hat{\mu} \mu \alpha \hat{\eta} \mu \alpha$ ). Voir les dialogues de Platon: Cratyle 400c, Phédon 33c, Gorgias 443a.

11. Pour une réfutation en règle de Bultmann, voir Gundry (1976), qui plaide pour un retour à une compréhension entièrement physique du corps, marqué par la mort. 
c'est de manière fluctuante, toujours pour régler des problèmes concrets et toujours dans la perspective du rapport à Dieu.

\section{Quelques mots et leur fonctionnement textuel}

Paul utilise principalement quatre mots pour parler de l'humain: $\psi v x \eta ́$ (vie animée, âme), $\sigma a ́ p \xi$ (chair, viande), $\sigma \omega \hat{\omega} \mu \alpha$ (corps) et $\pi v \in \hat{u} \mu \alpha$ (souffle,

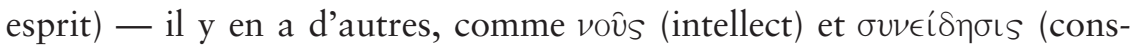
cience $)^{12}$. Il ne faut pas traduire trop mécaniquement ces mots, car ce ne sont pas des termes techniques, mais plutôt des figures sémantiques indéterminées qui prennent sens dans un texte, en relation l'une par rapport à l'autre, et qui n'ont pas la même valeur d'un texte à l'autre ${ }^{13}$. À l'aide de trois exemples, nous observerons comment ils fonctionnent dans des textes précis et comment ils font fonctionner ces textes. Auparavant, cependant, une brève remarque s'impose: le mot $\sigma a ́ p \xi$ ne désigne jamais a priori la sexualité, qui serait sinon dévalorisée et rejetée du côté du bestial; quant à $\pi v \in \hat{v} \mu \alpha$, je choisis de le traduire pour l'instant par souffle, et non par esprit, pour renvoyer à la métaphore originelle avec laquelle le texte construit sa signification. Je suis conscient que ce choix a pour conséquence de corporaliser le $\pi \nu \in \hat{v} \mu \alpha$ et de rendre plus difficile son interprétation intellectuelle, voire «spiritualiste». Chair, corps, souffle et à un degré moindre, vie animée (psychée) - renvoient tous à une matérialité pour mettre en discours l'humain.

Dernières remarques méthodologiques: le choix de nos trois textes, qui ne forment qu'un échantillon fort réduit, n'est pas arbitraire. La Première lettre aux Corinthiens est une lettre dont un des fils conducteurs, véritable thème transversal, est la réflexion sur le corps dans ses

12. La dernière synthèse complète remonte à trente ans: Jewett (1971). Auparavant, soulignons les œuvres majeures de deux homonymes: H.W. Robinson (1913) et J.A.T. Robinson (1952). Depuis ce dernier ouvrage, il existe une tendance à prioriser la question du corps; ainsi, encore récemment: Sandnes (2002), Son (2001) et Martin (1995).

13. "Words take their meaning fundamentally not from etymology nor even from dictionary, but from usage; [...] neither the Old nor the New Testament writers developed a specialized or technical, denotative vocabulary for theoretical discussion of the human person. And if this is so, then contemporary interpreters ought to exercise care when reading the biblical materials in light of specialized language that has developed subsequently. (Green 1999, 5) 
multiples acceptions (Guénel 1983). De plus, nous constaterons que dans la même lettre, plusieurs mises en discours de l'humain sont produites et placées en tension l'une par rapport à l'autre. Enfin, l'approche retenue est synchronique et se distingue d'une approche référentielle, rhétorique ou historique, qui cherche à reconstituer (de manière forcément hypothétique) les positions corinthiennes que Paul essaierait de corriger, ou à comparer les positions pauliniennes avec celles des philosophies grecques de l'époque (particulièrement le stoïcisme). Il s'agira plutôt d'une lecture du texte tel qu'il se présente, comme discours marqué par une énonciation, une argumentation, une succession d'images en tension, induisant certains repères anthropologiques ${ }^{14}$. Ce sera là la principale originalité exégétique de cet article - étant rappelé que l'auditoire visé n'est pas celui des spécialistes de la Bible (auxquels il apprendra peu), mais les autres collègues qui risquent avec moi le jeu de l'interdisciplinarité.

\subsection{Co 6,12-20: le corps entre la chair et le souffle}

(12) "Tout m'est permis ", mais tout ne profite pas. "Tout m'est permis ", mais je ne serai pas maîtrisé par quelque chose. ${ }^{(13)}$ Les aliments sont pour le ventre et le ventre pour les aliments, or Dieu abolira ceux-ci comme celui-là. Or, le corps n'est pas pour la fornication, mais pour le seigneur, et le seigneur pour le corps. ${ }^{(14)} \mathrm{Or}$, Dieu a relevé le seigneur et nous relèvera aussi, par sa puissance. ${ }^{(15)} \mathrm{Ne}$ savez-vous pas que vos corps sont des membres de Christ? J'enlèverais donc les membres du Christ pour en faire des membres de fornicatrice! Absolument pas! ${ }^{(16)}$ ou ne savez-vous pas que celui qui s'unit à la fornicatrice n'est [avec elle] qu'un unique corps? Car les deux, est-il dit, deviendront une chair unique. ${ }^{(17)}$ Mais celui qui s'unit au seigneur n'est [avec lui] qu'un unique souffle. ${ }^{(18)}$ Fuyez la fornication! Tout péché que fait l'humain est en dehors du corps, or, celui qui fornique pèche envers son propre corps. ${ }^{(19)} \mathrm{Ou}$ ne savez-vous pas que votre corps est un sanctuaire du souffle saint en vous, que vous avez de la part de Dieu? Et que vous ne vous appartenez pas? ${ }^{(20)}$ Car vous avez été achetés bien cher! Glorifiez donc Dieu dans/par votre corps.

14. Mon approche diffère donc de celle de Murphy-O'Connor (1978) qui affirme d'emblée: "The interpretation of 1 Corinthians is greatly conditioned by the exegete's assessment of the situation at Corinth, because Paul's words can mean different things when read against various backgrounds. » (391) 
Ce texte, où on retrouve les mots corps, chair, souffle, est fort curieux. Il s'agit d'une exhortation à ne pas fréquenter la prostituée ${ }^{15}-$ comportement dont l'apôtre a été informé et qui se réclamait peut-être d'un enseignement paulinien galvaudé par les Corinthiens: "Tout m'est permis ${ }^{16}$.» Or, ce propos anecdotique est l'occasion d'une «discussion

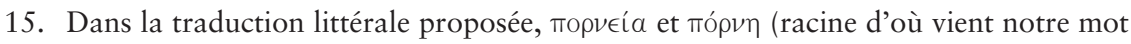
pornographie) ont respectivement été rendus par fornication et fornicatrice, afin de rendre la répétition de vocabulaire. Пópv $\eta$ est habituellement traduit par prostituée.

16. Пávта " $\xi^{\prime \prime} \in \sigma T \nu \nu$ revient aussi en 1 Co 10,23 , sous la même forme d'une double énonciation suivie chaque fois d'une correction marquée par $a \lambda \lambda \lambda^{\prime}$ oủ. L'hypothèse d'un slogan corrigé par Paul est donc plausible, d'où l'utilisation des guillemets dans la traduction. Par ailleurs, Murphy-O'Connor (1978) et Hays (1997), à partir de l'observation de la structure parallèle des v. 13-15 et des tensions du texte, proposent une lecture dialoguée du passage, comme confrontation de deux anthropologies, celle des Corinthiens qui dévalorisent le corps et justifient ainsi paradoxalement une sexualité désordonnée (v. 13a et 18a) et celle de Paul qui valorise le corps et maximalise l'importance de la sexualité (v. 13b-14 et 18b):

Corinthiens : - ${ }^{(12)}$ Tout m'est permis...

PAUL: - Mais tout ne profite pas!

- Tout m'est permis...

- Mais je ne serai pas maîtrisé par quelque chose!

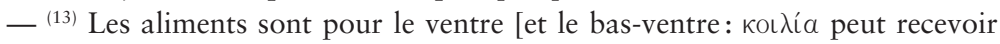
un sens génital, Senft 1990, 83] et le ventre pour les aliments: Dieu abolira ceux-ci comme celui-là.

- Or, le corps n'est pas pour la fornication, mais pour le seigneur, et le seigneur pour le corps: ${ }^{(14)}$ Dieu a relevé le seigneur et nous relèvera aussi, par sa puissance. $[\ldots]$

- ${ }^{(18)}$ Tout péché que fait l'humain est en dehors du corps...

- Or, celui qui fornique pèche envers son propre corps!

L'hypothèse est intéressante, mais demeure risquée comme l'indique lui-même Hays: "there is some guesswork involved in reconstructing this dialogue, because the ancient Greek manuscripts do not use quotation marks» (102). Or, Byrne (1983) a bien montré que couper en deux le v. 18 ne résolvait pas le problème de cohérence et que l'attribuer en entier à Paul faisait sens; le parallélisme structurel peut être attribué à Paul; le marqueur de transition or ( $\delta \dot{e}$, v. 13 b et 18) est moins fort que mais (ả $\lambda \lambda a ́$, v. 12) et peut indiquer une distinction ou une exception (Rosner 1995, 144). Bien plus, mon analyse montrera, avec d'autres commentateurs (par exemple Senft 1990; Conzelmann 1975), que le texte, tel qu'il est, se lit parfaitement. Bien évidemment, on obtient alors une toute autre interprétation anthropologique, peut-être plus contradictoire, mais aussi plus nuancée. 
anthropologique». Presque chaque phrase amène un nouvel argument d'ordre théologico-anthropologique, en même temps qu'un débrayage par rapport à ce qui précède (Senft 1990, 81). Outre la thèse initiale, éminemment paradoxale, j’y relève pas moins de six arguments, schématisés au tableau 1 (page suivante).

La thèse (v. 12) est formulée à la première personne: «je / moi», placé devant un choix. Ce «je» disparaît pour laisser place au ventre (v. 13), au corps (v. 13), à «nous » (v. 14), à « vous » (v. 15a), pour réapparaître (v. 15b) et s'éclipser à nouveau devant une série d'acteurs : celui qui s'unit à la prostituée (v. 16), celui qui s'unit au seigneur (v. 17), «vous» (v. 18), l'humain et son corps (v. 18), puis enfin encore «vous» et «votre corps». Au-delà du jeu de la communication entre Paul et les Corinthiens, il semblerait que ces termes soient d'une certaine manière interchangeables: il s'agit toujours d'un humain au prise avec une décision.

Le premier argument est une analogie où deux réciprocités sont comparées (v. 13-14). Mais les deux réciprocités ne fonctionnent pas de la même manière: aliments et ventre sont dans un rapport d'utilité (ce qui serait aussi le cas du rapport unidirectionnel corps / fornication, rapidement écarté, le corps ne devant pas servir à la débauche), tandis que corps et seigneur sont dans un rapport d'appartenance. Étrangement, le corps, destiné à la résurrection par sa relation au seigneur, y est dissocié du ventre et des aliments, destinés à la destruction (Wiéner 1983, 90). La sexualité n'est pas à situer au même plan que les besoins physiques. À cause du parallélisme entre $13 \mathrm{a}$ et $13 \mathrm{~b}$, on s'attendrait, au v. 14, à ce que Dieu relève le corps comme il a relevé le seigneur (Jésus). On trouve plutôt l'affirmation: "Dieu nous relèvera." En conséquence, on peut émettre l'hypothèse que le corps représente ici non pas l'être biologique, mais le Soi, dans son appartenance au Christ et dans sa capacité relationnelle (Conzelmann 1975, 111). La perspective et l'enjeu de cette relation entre le Soi et le Christ est la résurrection, qui se trouve donc au cœur de l'anthropologie paulinienne. Pour l'apôtre, l'humain est avant tout défini en fonction de cette relation.

Le second argument (v. 15) renforce l'idée d'appartenance, qui deviendra peu à peu le fil conducteur d'une argumentation qui semblait à première vue tirer dans toutes les directions. Si les corps sont des membres qui appartiennent au Christ, c'est qu'ils sont pensés non pas dans une perspective individuelle, mais collective, ou mieux, communautaire. 
Tableau 1. Argumentation de 1Co 6,12-20

\begin{tabular}{|c|c|c|c|}
\hline Verset & & gument & Description \\
\hline 12 & 0 & $\begin{array}{l}\text { Thèse paradoxale } \\
\text { répétée } 2 \text { fois } \\
\text { différemment: }\end{array}$ & $\begin{array}{l}\text { «Tout m'est permis } \gg \text { MAIS... } \\
\ldots \text { «tout ne me profite pas } \\
=\ll \text { je ne serai pas maîtrisé par quelque chose» }\end{array}$ \\
\hline $13 a$ & 1 & Analogie & $\begin{array}{l}\text { réciprocité: aliments } \Leftrightarrow \text { ventre, } \\
\text { voués à la destruction par Dieu } \\
\text { réciprocité: corps } \Leftrightarrow \text { seigneur, } \\
\text { voués au relèvement par Dieu } \\
\text { et non: corps } \Rightarrow \text { fornication }\end{array}$ \\
\hline 15 & 2 & $\begin{array}{l}\text { Savoir } n^{\circ} 7 \\
\text { Possibilité écartée }\end{array}$ & $\begin{array}{l}\text { corps }=\text { membres du Christ (appartenance) } \\
\text { membres du Christ } \Rightarrow \text { membres de prostituée }\end{array}$ \\
\hline $\begin{array}{l}16 a \\
16 b\end{array}$ & 3 & $\begin{array}{l}\text { Savoir } n^{\circ} 2 \\
\text { Réminiscence) } \\
\text { scripturaire } \\
(\text { Gn 2,24) }\end{array}$ & $\begin{array}{l}\text { union à la prostituée } \Rightarrow \text { un seul corps } \\
\text { deux } \Rightarrow \text { une seule chair }\end{array}$ \\
\hline $\begin{array}{l}17 \\
18 a\end{array}$ & & Impératif & $\begin{array}{l}\text { union au seigneur } \Rightarrow \text { un seul souffle } \\
\text { «Fuyez la fornication!» }\end{array}$ \\
\hline $18 b$ & 4 & Distinction & $\begin{array}{l}\text { péché } \Rightarrow \text { extérieur du corps } \\
\text { sauf fornication } \Rightarrow \text { corps }\end{array}$ \\
\hline $19 a$ & 5 & Savoir $n^{\circ} 3 a$ & Corps $=$ sanctuaire du souffle \\
\hline $\begin{array}{l}19 b-20 a \\
2 o b\end{array}$ & 6 & $\begin{array}{l}\text { Savoir } n^{\circ} 3 b \\
\text { Impératif }\end{array}$ & $\begin{array}{l}\text { Non appartenance, car rachetés } \\
\text { «Glorifiez Dieu!» }\end{array}$ \\
\hline
\end{tabular}

L'image, à peine esquissée, est forte (et encore une fois paradoxale): les corps font partie d'un Corps ${ }^{17}$. Implicitement, le texte pose donc la question au corps: à quel Corps appartiens-tu?

17. La métaphore corporelle pour désigner la communauté et son rapport au Christ reviendra en 1Co 12,12-30 - à ce sujet, Perrot $(2000,45)$ parle de «corporation du Christ ». Comparer 1Co 6,15: «vos corps sont des membres de Christ » à 1Co 12,27: «vous êtes, vous, le corps de Christ, et membres chacun pour sa part». 
Le troisième argument (v. 16-18a), où la même équation $(1+1=1)$ est attribuée successivement au corps, à la chair et au souffle, donne vraiment l'impression d'un glissement à chaque étape du raisonnement.

- Au v. 16, la gravité de la prostitution, comme acte sexuel illégitime, est démontrée de manière ironique par une citation scripturaire implicite, tirée de Gn 2,24. L'union légitime de l'homme et de la femme est voulue par Dieu, contrairement à la fornication. Mais en citant l'écriture, le texte paulinien passe de l'image des corps qui n'en forment qu'un... à l'image de l'homme et de la femme qui ne forme qu'une seule chair. Faut-il en conclure que les deux mots $\sigma \omega \hat{\mu} \mu \alpha$ et $\sigma a ́ p \xi$ sont ici équivalents ? Laissons la question ouverte. De toute manière, en traduisant en Gn 2,24 le mot hébreu

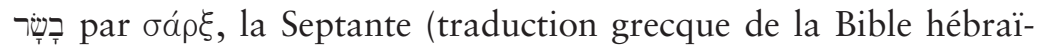
que) donne à ce dernier mot une valeur neutre.

- Or, au v. 17, l'union somatique (ou charnelle?) avec la prostituée est opposée à l'union spirituelle avec le seigneur. Pourquoi ce changement de vocabulaire? Est-ce seulement parce que l'union avec le Christ ne pouvait être ramenée à l'union sexuelle (qu'elle soit illégitime ou légitime)? Il faut bien voir ici que $\pi v \in \hat{u} \mu \alpha$ s'oppose antithétiquement soit à $\sigma \omega \hat{\mu} \mu$ (si on passe directement du

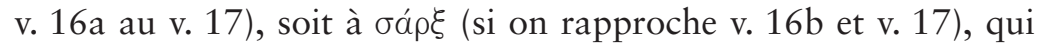

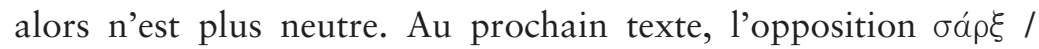
$\pi \nu \in \hat{v} \mu a$ sera plus clairement établie, tandis que l'image du souffle comme espace de relation (non plus entre le croyant et Christ, mais entre le croyant et Dieu) recevra une description moins laconique.

On est donc passé de corps à chair, puis à souffle, et nous avons pu construire une équivalence et deux oppositions : corps = chair, $\operatorname{corps} \neq$ souffle, chair $\neq$ souffle. Rendu à ce stade, nous rencontrons le premier de deux impératifs, formulé négativement: «Fuyez la fornication.» (v. 18a)

Les trois derniers arguments sont encore plus syncopés que les précédents. Le quatrième (v. 18b) énonce que la fornication est un péché contre son propre corps et non extérieur au corps. Serait-il exagéré de comprendre: un péché envers soi-même, non extérieur à soi, et non dirigé vers les autres? Ou encore, selon la thèse de Byrne (1983), que la fornication inhibe la fonction relationnelle du corps? Le cinquième argument (v. 19a) est une métaphore où il est dit que le souffle saint (de Dieu) 
habite en «vous », c'est-à-dire dans "votre corps» comparé à un temple $^{18}$. Dans ces deux arguments, le corps fonctionne comme l'équivalent du Soi, ce qui renforce notre hypothèse du v. 14.

Enfin, le sixième argument (v. 19b-20) ramène l'idée d'appartenance,

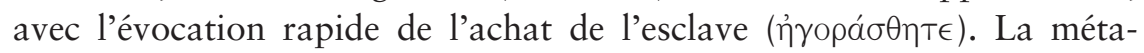
phore, importante chez Paul, s'inscrit dans le cadre socio-culturel de son époque et peut être interprétée de deux manières. D'une part, comme l'esclave qui a été affranchi et qui, même libre, dépend du maître qui l'a libéré en payant la rançon, le chrétien est libre, mais finalement... appartient à son seigneur. À titre d'affranchi, il est agrégé à la clientèle de son illustre patron ${ }^{19}$. D'autre part, on pourrait faire une lecture plus radicale (et plus paradoxale encore) de l'affirmation «vous ne vous appartenez

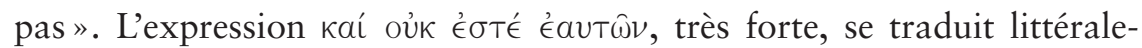
ment: "vous n'êtes pas à vous-mêmes » et évoque le statut juridique de l'esclave, propriété mobilière de son maître, qui en fait ce qu'il veut. De manière métaphorique, le corps du chrétien appartient à son maître, Dieu $^{20}$ - la métaphore signifiant aussi que Dieu n'est pas comme un maître, puisque lui être assujetti, c'est trouver la liberté21.

18. Ailleurs dans la même lettre, Paul a déjà appliqué l'image du temple à la communauté - ce qui montre comment il est facile de passer du corps individuel au Corps communautaire: "Ne savez-vous pas que vous êtes un sanctuaire de Dieu, et que le souffle de Dieu habite en vous?" (1Co 3,16)

Dans un autre ordre d'idées, s'il est clair que le souffle (qualifié de saint) vient de Dieu, il pourrait être possible ici de lire que le corps lui aussi vient de Dieu. On rapporte généralement la proposition relative «que vous avez de la part de Dieu » au souffle saint (Bible de Jérusalem, TOB), mais il n'est pas exclu grammaticalement que la proposition puisse qualifier l'origine du corps (Segond et ma traduction conservent cette ambiguïté). Ceci en lien avec la fin du verset: "vous ne vous appartenez pas». Ailleurs en 1Co 7,40, on retrouvera l'idée d'avoir le souffle de

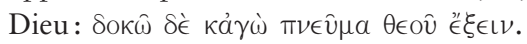

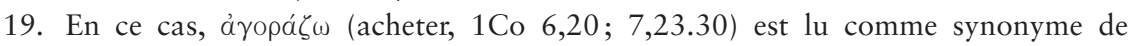
$\epsilon^{\epsilon} \xi a \gamma o \rho a ́ \zeta \omega$ (racheter, Ga 3,$13 ; 4,15$ ), ce qui ne va pas de soi.

20. Glancy (1998) développe une interprétation en ce sens, en soulignant toutefois que les Corinthiens chrétiens, homme ou femme, qui seraient esclaves d'un maître non chrétien (hypothèse fortement plausible, lire 1Co 1,27-28 sociologiquement), risquent d'être coincés entre l'impératif d'obéir à Dieu et celui de consentir aux caprices sexuels de leur maitre.

21. Rappelons que Ricœur $(1975,11)$ insiste justement sur la fonction identificatrice, en même temps que négatrice, de la métaphore - une remarque essentielle trop souvent négligée en ce qui concerne la Bible; ainsi, il faudrait dire: «Dieu est père et il n'est pas père. » 
Quoi qu'il en soit de la lecture qu'on adopte, il faut noter la transformation spectaculaire entre le v. 12 et le v. 20. On passe de l'affirmation: "je ne serai pas maîtrisé par quelque chose» qui implique la possibilité (et même la nécessité) d'un choix, à l'affirmation: "vous ne vous appartenez pas ", qui clôt la discussion éthique sur une obligation. Dit autrement: le v. 12 souligne une liberté (nouvellement) acquise et qu'il ne faudrait pas gaspiller, tandis que le v. 20 indique l'absence de liberté2 ${ }^{2} \ldots$

En résumé, du point de vue anthropologique, retenons que le corps semble fonctionner comme l'équivalent du Soi, placé devant une alternative: s'assujettir à la chair, finalement comprise comme s'opposant à Dieu, ou respecter son appartenance au seigneur, en demeurant dans l'ordre du souffle. S'unir à la prostituée n'est pas un geste banal mais implique un acte relationnel intense et décisif, qui engage totalement le Soi... en réduisant sa capacité relationnelle: la prostitution est une non-relation (voir Byrne 1983). La question du texte n'est pas de spéculer sur la structuration de l'être humain, mais de réfléchir à son comportement, dans l'optique de sa relation à Dieu, non pas tant dans une perspective individuelle que communautaire. Le dernier impératif du texte, "Glorifiez

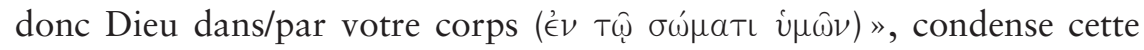
intuition: nous sommes en présence d'un Soi corporel, interpellé collectivement, au pluriel, dont le corps est le lieu ou l'instrument de glorification de Dieu. Une discussion éthique fort concrète sera donc devenue une porte d'entrée sur l'anthropologie de Paul.

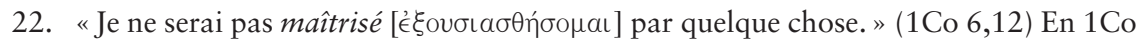
7,4 , dans une autre discussion sur la sexualité — cette fois, la vie conjugale —, Paul

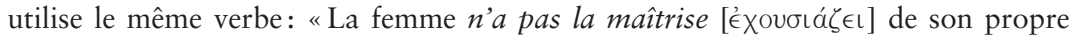
corps mais l'homme; semblablement, l'homme n'a pas la maitrise de son propre corps, mais la femme.» Une rencontre sexuelle vraiment relationnelle est correcte et implique la réciprocité et une perte de contrôle. 


\subsection{Co 2,10-3,4: trois rapports à Dieu (spirituel, psychique, charnel)}

${ }^{(10)}$ En effet, c'est à nous que Dieu [le] dévoila par le souffle. Car le souffle sonde tout, même les profondeurs de Dieu. ${ }^{(11)}$ Qui donc parmi les humains sait ce qui est dans l'humain, sinon le souffle de l'humain qui est en lui? De même, les [choses] de Dieu, personne ne les connaît, sinon le souffle de Dieu. ${ }^{(12)}$ Pour nous, nous ne reçûmes pas le souffle du monde, mais le souffle qui vient de Dieu, afin que nous sachions les [choses] accordées gracieusement par Dieu. ${ }^{(13)}$ Et nous en parlons, non pas par les paroles qu'enseigne la sagesse humaine, mais par [celles] qu'enseigne le souffle,

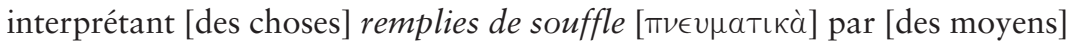

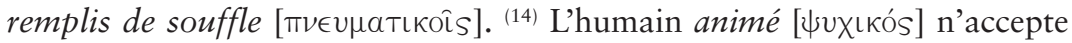

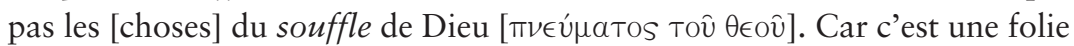
pour lui et il ne peut le connaître, parce que c'est à la manière $d u$ souffle

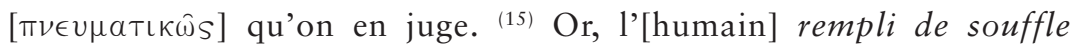

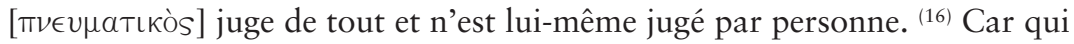
a connu la pensée du Seigneur pour l'instruire? Or nous, nous avons la pensée du Christ.

${ }^{(3,1)}$ Pour moi, frères, je n'ai pu vous parler comme à des [gens] remplis de souffle, mais seulement comme à des charnels (баркívoıs), comme à des nourrissons en Christ. ${ }^{(2)}$ C'est du lait que je vous ai fait boire, non de la nourriture solide: vous ne l'auriez pas supportée. Mais vous ne la supporteriez pas davantage aujourd'hui, ${ }^{(3)}$ car vous êtes encore charnels. Puisqu'il y a parmi vous jalousie et querelles, n'êtes-vous pas charnels et ne marchez-

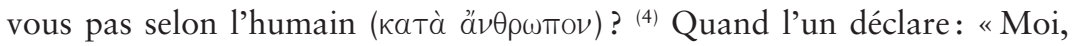
j'appartiens à Paul ", l'autre: "Moi à Apollos », n'êtes-vous pas humains?

L'extrait ci-dessus est délimité quelque peu artificiellement, suivant la logique du vocabulaire "anthropologique» qui nous intéresse. De fait, le discours en «nous » commence en 2,6, formant un débrayage par rapport à un discours au «je » en 2,1-5, et se termine en 2,16, pour faire place ensuite au rembrayage du «je» en 3,1-5. Des répétitions confirment ce découpage: (1) nous parlons apparaît aux v. 6.7 et 13, formant presque une inclusion; (2) connaître (en caractère gras ci-dessus) revient cinq fois à titre de thématique transversale (v. 8.8.11.14.16). Conzelmann (1975, 57) distingue néanmoins dans cette section deux paragraphes: v. 6-9 et v. 10-16 - ce dernier paragraphe étant délimité lui-même par une question. On obtient la structure suivante: 
2,1-5 «Ma parole (v. 4)

2,6-16 Dévoilement du mystère

«Nous» parlons (v. 6.7)

\1: v. 6-9: sagesse de ce monde et sagesse de Dieu

\2: v. 10-16: souffle de ce monde et souffle de Dieu

- Qui donc parmi les humains connaît ce qui est dans l'humain? (v. 11)

- Qui a connu la pensée du Seigneur pour l'instruire? (v. 16)

« Nous » parlons (v. 13)

3,1-4 «Je» n'ai pu vous parler (v. 1)

Cette précision de délimitation et de structure s'avère importante en regard du contexte apocalyptique ${ }^{23}$ qu'elle confère à 1Co 2,6-3,5. Le discours de Paul sur la croix est discours apostolique de dévoilement

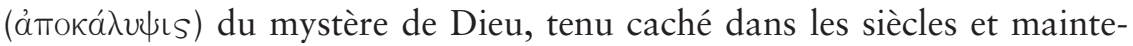
nant manifesté, grâce à l'expérience du souffle (Hays 1997, 43). En Jésus se dévoile l'irruption d'un monde nouveau (et donc d'une humanité nouvelle), appelé à remplacer le monde ancien (et donc la vieille humanité). Le passage du «je» au "nous» marque, entre autres, la solennité de ce dévoilement et le statut privilégié qu'il confère à l'apôtre, avec l'autorité qui l'accompagne.

Les derniers versets (3,3-4) donnent l'ancrage concret de la discussion et renvoient à un problème soulevé en 1Co 1,10-16. Dans la jeune, tumultueuse et peu nombreuse communauté de Corinthe, il existe déjà au moins quatre clans qui se réclament respectivement de Paul, Apollos, Pierre et Christ - encore ici, il s'agit d'une question d'appartenance: où vous situez-vous? Avant de pouvoir répondre à cette question (par le texte ici étudié), Paul développe le discours de la croix, marqué par la faiblesse et la folie, qui s'oppose donc, d'une part au discours juif de la puissance des signes, d'autre part au discours grec de la sagesse (1Co 1,17-25, voir Badiou 1998, 43-57). Preuve (un peu sarcastique) en est que les Corinthiens choisis par Dieu sont l'exemple même de la faiblesse et de la folie (1Co 1,26-31), de même que Paul lui-même, «faible, craintif

23. Au début de 1Co 2, on observe l'accumulation du vocabulaire technique apocalyptique: mystère de Dieu (v. 1), sagesse et princes de cet âge (v. 6.8), sagesse mystérieuse, cachée, destinée d'avance, avant les âges (v. 7), dévoiler (v. 10). 
et tout tremblant », qui a pourtant reçu et transmis la révélation de ce discours d'un Seigneur crucifié (1Co 2,1-9 $)^{24}$.

Par leurs querelles et leur jalousie, les Corinthiens ont un comportement humain, infantile, charnel, qui s'oppose à un comportement rempli de souffle ou, en un mot, spirituel. En 3,3, le кaтà ä $\nu \theta \rho \omega t o v$ semble donc

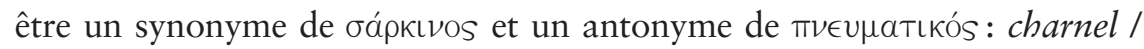
humain s'oppose à rempli de souffle. En ce cas, faudrait-il traduire charnel par «condition humaine ${ }^{25}$ » ? Or, en 2,14, on observe plutôt l'oppo-

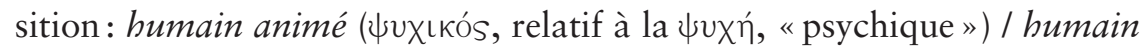
rempli de souffle ( $\pi \nu \in v \mu a \tau i \kappa o s$, relatif au $\pi \nu \in \widetilde{v} \mu \alpha$, «spirituel»). Il existe deux humanités. À nouveau, on constate dans un même texte un flottement dans l'emploi des termes, et des dichotomies successives au fil de l'enchaînement du discours. De fait, le texte construit une série d'oppositions qui ne s'alignent pas à la perfection:

$\begin{array}{ll}2,11 & \text { souffle de l'humain } \\ 2,12 & \text { souffle du monde } \\ 2,13 & \text { discours de la sagesse humaine } \\ 2,14-15 & \text { humain animé («psychique») } \\ 3,1 & \text { humains charnels } \\ 3,2 & \text { lait }\end{array}$

souffle de Dieu souffle de Dieu discours du souffle humain rempli de souffle («spirituel») humains remplis de souffle nourriture solide

De manière globale, on comprend que le comportement charnel des Corinthiens (qui n'a rien à voir ici, répétons-le, avec la sexualité), ou encore leur perspective "psychique ", est en opposition au comportement voulu par Dieu ou attendu des humains remplis de son souffle. Il est significatif que ce soit à nouveau à l'occasion d'une discussion éthique (à propos d'une division communautaire) que nous glanons quelques informations anthropologiques. Dans ce qu'il est, corps, souffle, chair, vie animée, l'humain doit faire des choix.

Certes, animé s'oppose à rempli de souffle, puis charnel s'oppose à rempli de souffle, mais les choses ne sont pas si simples. Car on découvre ici trois sortes de souffle: celui de Dieu, de l'humain et du monde, qui

24. Pour une analyse sociocritique du texte, qui vise à reconstituer le profil de la communauté à partir des données inscrites dans la rhétorique paulinienne, voir Theissen 1996 (allemand 1975).

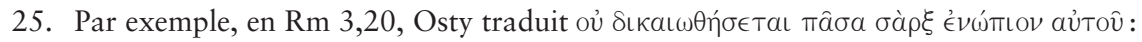
«Aucun homme [littéralement: pas... toute chair] ne sera justifié devant lui.» 
sont des médiateurs de connaissance. Si dans la colonne de droite nous avons un unique mot: souffle (de Dieu), dans la colonne de gauche on retrouve souffle du monde, sagesse humaine, psychique, charnel. Une manière de s'y retrouver consiste à envisager les deux colonnes selon la dichotomie apocalyptique déjà évoquée: le monde ancien est celui de la chair et de la vie animée, le monde nouveau est celui du souffle. Les Corinthiens sont invités à faire le passage de l'un à l'autre.

La mise en discours ne décrit pas uniquement le souffle en termes dichotomiques, mais aussi en termes fonctionnels. Répétons-le: les verbes de connaissance $\gamma \iota \nu \omega ́ \sigma \kappa \omega$ et oîfa reviennent cinq fois (connaître et savoir, en caractère gras dans le texte ci-haut). Le souffle de Dieu connaît Dieu; le souffle humain connaît l'humain; en recevant le souffle de Dieu, l'humain peut connaître Dieu et ses dons de grâce, ce qu'il ne saurait faire avec le souffle du monde, puisque le souffle (de Dieu) s'oppose aussi à la sagesse humaine. Le souffle devient l'interface où l'humain et le divin se rejoignent. Le souffle de l'humain est sa capacité de connaître Dieu, ou d'entrer en contact avec lui.

En redonnant à $\pi v \in \hat{v} \mu \alpha$ son sens métaphorique de souffle (vent, respiration) et en examinant son fonctionnement en 1Co 2,10-3,4, je l'interprète non plus selon un dualisme matériel / spirituel-incorporel, mais selon un registre de part en part matériel et corporel. Le souffle est cette force en moi qui m'habite et me fait vivre, plus intime à moi que moi-même, telle ma respiration; il est aussi cette atmosphère qui m'entoure, où je baigne et je puise pour alimenter ma respiration. Analogiquement, le souffle de Dieu est son intimité (sa respiration) et sa puissance, qui nourrit, entoure et remplit le croyant. Il n'est d'ailleurs pas anodin que la pratique spirituelle de la prière, dans toutes les religions, soit liée d'une manière ou d'une autre à la respiration: le souffle du divin se mélange au souffle humain, au point où il devient difficile de distinguer l'un et l'autre. Enfin, à partir de la mise en discours du texte, on pourrait ajouter que l'air respiré par les Corinthiens n'est pas pur oxygène divin, puisqu'ils respirent encore aussi, de toute évidence, le souffle du monde où ils sont immergés, d'un monde incapable de connaître Dieu et d'apprécier le discours de la croix.

Une dernière remarque concerne la hiérarchisation suggérée par le texte. Certes, toute dichotomie comporte une hiérarchie implicite. Mais le discours paulinien insiste indubitablement sur la supériorité de ceux qui sont remplis du souffle. D’abord, cet énoncé péremptoire: «L'[humain] 
rempli de souffle juge de tout et n'est lui-même jugé par personne» $(2,15)$; ensuite, l'infantilisation des Corinthiens ramenés au statut de bébés allaités $(3,1-2)$. Or, cette hiérarchie n'est pas interne à l'humain, du genre de celle qu'on retrouve chez Platon, mais compare divers types humains: les spirituels d'un côté, les psychiques et charnels de l'autre.

\subsection{Co 15,35-53: corps psychique et corps spirituel}

Le troisième texte que nous examinons maintenant constitue le sommet de la réflexion de 1Co sur le corps. Après le corps communautaire divisé (1Co 1-4 et 12-14), le corps qui peut appartenir à la prostituée ou au Christ (1Co 6,12-20) et le corps eucharistique du Christ partagé comme pain et vin (1Co 10-11), 1Co 15 met en discours le corps ressuscité. Le tableau 2 (page 115) présente une traduction structurée qui s'inspire de l'analyse de Normand Bonneau (1993) et qui nous permettra de mieux saisir l'anthropologie paulinienne ${ }^{26}$. Pour alléger la traduction ainsi que

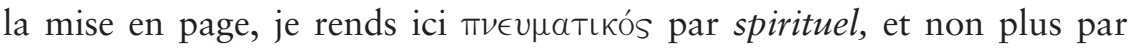
rempli de souffle (comme en 1Co 2,10-3,4); de même, భuxıкós est rendu par psychique, et non animé.

Paul répond à la question «avec quels corps les morts ressuscitentils [litt.: se relèvent-ils] ${ }^{27}$ ?» (v. 35b) D'abord à l'aide de deux analogies de son cru, qui empruntent à des registres botaniques, zoologiques et astronomiques (Martin 1995), mais qui sont peut-être inspirées d'un enseignement pharisien (Morissette 1972a, 1972c). Ensuite, sa réponse s'appuie sur un argument scripturaire, lorsqu'il relit Gn 2,7 de manière midrashique. Les deux analogies construisent successivement deux contrastes (v. 36-41), pour ensuite les télescoper (v. 42-44): sur un plan temporel qui implique une continuité, le corps à venir découle du corps présent (v. 36-38); sur un plan spatial qui implique une discontinuité, le corps à venir est supérieur au corps actuel (v. 39-41). L'argument scripturaire complète ce télescopage continuité / discontinuité. D'un bout à

26. Pour une étude de ce texte sous l'angle de la résurrection proprement dit et une bibliographie plus étoffée, voir Gignac (2001, 291-299), Asher (2000) et Martin (1995).

27. Encore une fois, il n'est pas tenu compte ici des éventuelles conceptions corinthiennes que le texte paulinien entendrait corriger. Pour une approche en ce sens, voir plutôt Martin (1995). 
l'autre du texte, le pivot de la discussion est le corps, qu'il soit végétal (v. 38), céleste (les astres, v. 41) ou terrestre (les animaux, v. 39), ou encore psychique ou spirituel (v. 44b).

Deux oppositions structurent le texte. De manière étonnante, et contrairement à ce qu'une lecture rapide pourrait suggérer, il ne s'agit pas de l'opposition que nous avons rencontrée en 1Co 6,12-20 et 1Co 3,1-4 entre chair et souffle. Premièrement (v. 39-41), le terme chair s'oppose à

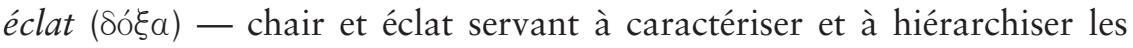
différentes catégories de "corps animé" (doué d'une "âme»): corps humain, animal, astral. À première vue, chair est donc neutre ici; pourtant, le contraste entre $\sigma a ́ p \xi$ et $\delta o ́ \xi a$ implique un jugement de valeur,

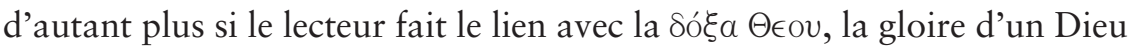
qui est au ciel. Deuxièmement (v. 42-44), le corps psychique ( $\sigma \omega \dot{\mu} \mu a$

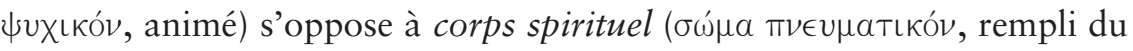
souffle), qui lui est donc supérieur. Cette opposition psychique / spirituel avait surgi en 1Co 2,10-16. De même que les chairs sont hiérarchisées entre elles (de l'humain au poisson) et que les astres n'ont pas la même brillance (du soleil aux étoiles), de même le corps ressuscité qualifié de spirituel sera supérieur au corps actuel qualifié de psychique.

On est loin du dualisme corps / âme: de chaque côté de la mort, il y a un corps; la భuxý n'est pas au-delà de la mort, mais en deçà ; elle est par ailleurs inférieure au souffle $(\pi \nu \in \hat{u} \mu \alpha)$. Le contraste s'établit plutôt entre les forces qui habitent le corps de part et d'autre de la mort: dans un cas la vie fragile, dans l'autre cas la force même de Dieu. Psychique est ici qualifié par les mots corruptibilité, mépris, faiblesse, des traits qu'on attribuerait plutôt spontanément à charnel, tandis que spirituel est qualifié par association avec l'incorruptibilité, l'éclat, la puissance. Le contraste se prolonge dans le rappel scripturaire de Gn 2,7 qui oppose le premier Adam et le dernier Adam (pour une analyse détaillée, voir Gignac 2001, 296-298). Le premier Adam, par son caractère psychique,

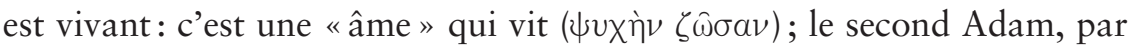
son caractère spirituel, donne la vie: c'est un souffle qui fait vivre (

Nous retrouvons ici une vision apocalyptique, dont la résurrection est un motif associé, puisque celle-ci constitue la manifestation de cet âge nouveau qui prolonge et remplace l'ancien. Le corps animé se transforme: du réceptacle passif de la vie terrestre (comme Adam en Genèse), il devient l'agent de la vie divine, céleste, sans commune mesure avec la 


\section{Tableau 2. Texte structuré de 1Co 15,26-49}

\begin{tabular}{|c|c|c|}
\hline \multicolumn{3}{|c|}{ ARGUMENT PAR ANALOGIE } \\
\hline \multicolumn{3}{|c|}{ - exemple $1:$ avant / après (plantes) } \\
\hline \multicolumn{3}{|c|}{$\begin{array}{l}36 \text { Sot ! Ce que tu sèmes, toi, n'est vivifié s'il ne meurt. } \\
37 \text { Et ce que tu sèmes, ce n'est pas le corps à venir, mais un simple } \\
\text { grain, par exemple, de blé ou de quelque autre [plante] ; } \\
38 \text { or, Dieu lui donne le corps comme il a voulu, } \\
\text { à chacune des semences son propre corps. }\end{array}$} \\
\hline \multicolumn{3}{|c|}{ - exemple 2 : en bas (moins) / en haut (plus) (animaux = corps) } \\
\hline & $\begin{array}{l}\text { Toutes les chairs ne sont pas les mê } \\
\text { mais autre est celle des hommes } \\
\text { autre la chair du bétail, } \\
\text { autre la chair des oiseaux, } \\
\text { autre celle des poissons. }\end{array}$ & mes chairs, \\
\hline & $\begin{array}{l}\text { [ll y a] aussi des corps célestes et de } \\
\text { mais autre est l'éclat des céleste } \\
\text { autre celui des terrestres. }\end{array}$ & $\begin{array}{l}\text { s corps terrestres, } \\
\text { s, }\end{array}$ \\
\hline & $\begin{array}{l}\text { Autre l'éclat du soleil, } \\
\text { autre l'éclat de la lune, } \\
\text { autre } \overline{\overline{\text { l'éclat }}} \text { des étoiles. }\end{array}$ & \\
\hline Une & ile même diffère en éclat d'une étoile. & \\
\hline \multicolumn{3}{|c|}{ - synthèse } \\
\hline & $\begin{array}{l}\text { en est-il du relèvement des morts: } \\
\text { on est semé dans la corruptibilité, } \\
\text { on est semé dans le } \underline{\underline{\text { mépris, }}} \\
\text { on est semé dans la } \underline{\underline{\text { faiblesse, }}} \\
\text { on est semé corps psychique, }\end{array}$ & $\begin{array}{l}\text { on est réveillé dans l'incorruptibilité ; } \\
\text { on est réveillé dans l'éclat }\end{array}$ \\
\hline
\end{tabular}

\section{ARGUMENT SCRIPTURAIRE}

S'il y a un corps psychique,

45 C'est ainsi qu'il est écrit :

Le premier homme, Adam, parut en $\underline{\underline{a ̂ m e}}\left(\psi v \chi \eta^{\prime}\right) \underline{\underline{q u i} \text { vit }}$;

46 mais le psychique,

Le premier homme (est) tiré du sol, terrestre ;

il y a aussi un corps spirituel. Tel le terrestre, tels aussi les terrestres;

tel le céleste, tels aussi les célestes.

Et de même que nous avons porté l'image du terrestre,
$36(-) 37(-) / 38(+)$ verbes d'actions sujets personnels (tu + Dieu)

v. 39 ( $4 \times$ chair + autre) // v. 41 (4 x éclat + autre) (dans un ordre décroissant) un seul verbe (d'état)

Fusion de la perspective horizontale temporelle (verbes) et verticale spatiale qualitative (noms)

Reprise de l'intégration de la double transformation : temporelle et spatiale 
vie terrestre, parce que rempli de souffle. D'ailleurs, les versets qui suivent notre texte utilisent l'imagerie apocalyptique:

${ }^{(51)}$ Voici, je vous dis un mystère: nous ne nous endormirons pas tous, mais tous nous serons changés. ${ }^{(52)}$ En un instant, en un clin d'oeil, à la dernière trompette; car on sonnera la trompette, et les cadavres seront réveillés incorruptibles, et nous, nous serons changés. ${ }^{(53)}$ Il faut, en effet, que ce corruptible revête l'incorruptibilité, que ce mortel revête l'immortalité.

Dans l'argumentation du texte, il est notable que sur terre ou dans le ciel, qu'il soit psychique ou rempli de souffle, le corps (et donc l'humain) est toujours décrit en termes matériels (Martin 1995, 128). En recoupant 1Co 15,26-49 avec nos observations de 1Co 2,10-3,4, il appert que les chrétiens "avancés » expérimentent déjà, selon Paul, ce à quoi peut ressembler un "corps rempli de souffle", puisque leur souffle humain a déjà part au souffle divin. Peut-être Martin $(1995,128)$ a-t-il raison d'affirmer: "For Paul, the current human body is made up of sarx, psyche, and pneuma. The resurrected body will shed the first two of these entities-like so much detritus-and retain the third, a stuff of a thinner, higher nature.»

\section{Récapitulation des résultats}

Quelques conclusions se dégagent de nos observations textuelles:

1. Chacun des termes avec lesquels jongle Paul peut désigner le Soi sous différents aspects - je me situe en partie ici dans un certain

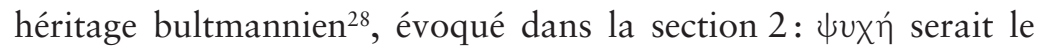

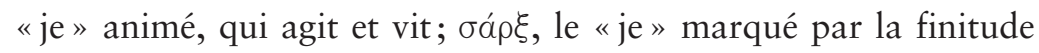
et le mal, éventuellement en opposition à Dieu; $\pi \nu \in \hat{v} \mu \alpha$, le «je» en relation avec Dieu; $\sigma \hat{\omega} \mu \alpha$, le «je» neutre physique, qui doit choisir entre chair et souffle, qui possède une capacité relationnelle et qui n'est pas compris comme pure individualité, mais aussi en lien avec un Corps, le collectif de solidarité des croyants.

28. Déjà, Käsemann $(1964,129)$ écrivait: «The individual concepts of Pauline anthropology are not intended to designate different parts of man, but the whole man with his various possibilities and relationships; thus these concepts are frequently interchangeable with "I"." 
2. Deux oppositions principales, dont le corps semble être l'enjeu ou le prétexte, ont été identifiées dans des textes pourtant tirés de la même lettre: chair et souffle (1Co 6,12-20 et 2,10-3,4), vie animée et souffle (1Co 2,10-3,4 et 1Co 15,35-53).

3. En corollaire des deux conclusions qui précèdent, il faut affirmer, avec la majorité des exégètes actuels, que les textes pauliniens se prêtent plus à une anthropologie holistique qu'à une anthropologie dualiste. Bien plus, on constate qu'avant ou après la mort, le vocabulaire est toujours d'une certaine façon "matérialiste». Dans une discussion entre neurosciences, phénoménologie et théologie, cela peut éventuellement servir de point de départ: «je» est un corps, être complexe mais matériel, multidimensionnel et non pas unidimensionnel, qui doit se situer face aux autres et à Dieu. Le Soi paulinien est éthique et destiné à la résurrection.

4. Le langage paulinien est métaphorique, cherchant à dire une intuition indicible (par sa nature et par la pauvreté du vocabulaire théologique dont l'apôtre dispose): la situation humaine dans un monde de finitude en relation avec Dieu. Pour paraphraser la citation de Vergote placée en exergue à cet article: ce n'est pas tant «je » qui réussit à se dire, que le langage (la déclinaison et l'articulation de corps, chair, vie animée et souffle) qui dit ce Soi.

5. Surtout, d'un texte à l'autre, on retrouve à l'arrière plan une vision apocalyptique du monde, selon laquelle le monde de Dieu est advenu en Jésus, remplaçant le monde actuel. La figure 1 schématise cette perspective. Ayant reçu le souffle, l'humain, corps vivant, est témoin de l'irruption de l'âge nouveau appelé à remplacer l'ancien. Cet âge nouveau est celui du souffle, qui ouvre une époque nouvelle (ère), un espace nouveau (aire) et qui constitue le milieu de vie, l'atmosphère vivifiant et spirituel où baigne le chrétien (air). Néanmoins, encore situé à l'intersection des deux mondes, l'humain éprouve un tiraillement entre un comportement charnel, opposé à Dieu, et un comportement conforme au souffle, en relation avec Dieu. Avant tout, l'humain est donc un «je» qui doit choisir - d'où l'importance que prendra le mot conscience ( $\sigma v v \in i ́ \delta \eta \sigma \iota s)$, dont nous n'avons pas parlé, mais que le corpus paulinien emploie de manière originale (quatorze apparitions dont huit en 1Co 8,7.10.12; 10, 25.27.28.29). 
Figure 1. L'irruption apocalyptique du monde nouveau

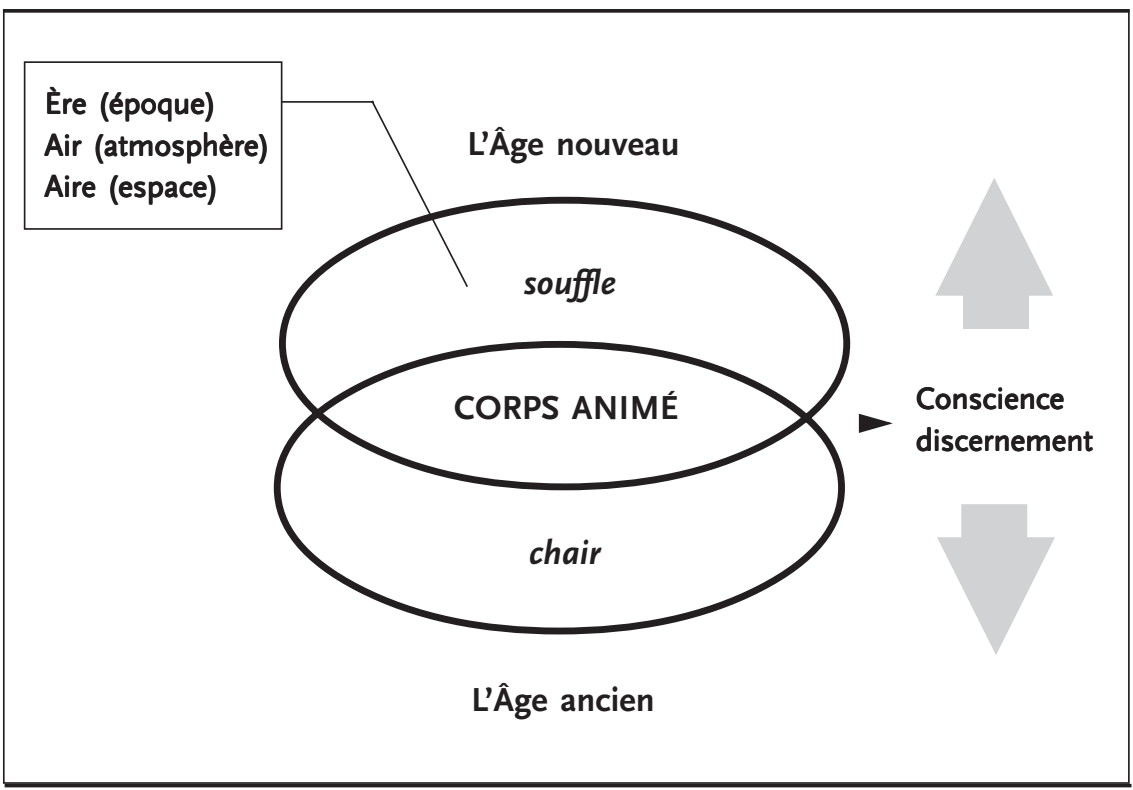

Ces quelques conclusions n'ont pas la prétention d'épuiser la question de l'anthropologie paulinienne. D'autres textes seraient à considérer, qui viendraient les nuancer. J'ai simplement voulu montrer que le vocabulaire paulinien n'est pas systématique mais s'inscrit dans le fonctionnement de textes particuliers. Or il se pourrait même que la piste du vocabulaire ne soit pas la plus intéressante. Au delà du problème des "composantes» ou plutôt, dans ma perspective, au delà des "aspects " de l'être humain, il y a chez Paul l'émergence du «sujet» (Vouga 2000). D'abord il y a cet apôtre qui, l'un des premiers dans l'antiquité, ose écrire en «je » et exposer, bien que toujours pudiquement, son cheminement et combat intérieur (voir Ga 1-2; Ph 3). Mais ensuite, il y a ce "corpus» paulinien qui convoque constamment le lecteur à devenir un sujet éthique et croyant. Dans cette ligne, le célèbre texte de Rm 7,7-25 ( «je ne fais pas ce que je veux...») serait à reprendre, non pas sous un biais référentiel, 
très (trop) débattu ${ }^{29}$, mais selon son fonctionnement textuel: comment ce «je» jamais identifié construit-il l'identité du lecteur?

Voilà une bonne question à poser aux neurosciences: y a-t-il une place, dans la discussion, ou du moins à l'horizon des recherches expérimentales, pour cette problématique d'un sujet qui s'interroge face à luimême (identité), face aux autres (éthique) et face à l'Absolu (espérance)?

\section{Références}

Agamben, G. (2000) [italien 2000], Le temps qui reste. Un commentaire de l'épitre aux Romains / trad. par J. Revel, Paris, Rivages.

Asher, J.R. (2000), Polarity and Change in 1 Corinthians 15: A Study of Metaphysics, Rhetoric, and Resurrection, Tübingen, Mohr Siebeck (Hermeneutische Untersuchungen zur Theologie 42).

Badiou, A. (1998) [1997], Saint Paul: la fondation de l'universalisme, Paris, Presses universitaires de France (Les Essais du Collège international de philosophie).

Blais, M. (1990), L'autre Thomas d'Aquin, Montréal, Boréal.

Bonneau, N. (1993), "The Logic of Paul's Argument on the Resurrection Body in 1 Cor 15:35-44a", Science et esprit, 45, p. 79-92.

Bultmann, R. (1951) [1955] [allemand 1948 (1953)], Theology of the New Testament / trad. par K. Grobel, New York, Scribner's.

— (1969) [allemand 1957], "Une exégèse sans présupposition est-elle possible?", dans Foi et Compréhension, t. II, Paris, Seuil, p. 167-175.

Byrne, B. (1983), «Sinning against One's Own Body: Paul's Understanding of the Sexual Relationship in 1 Corinthians 6:18 ", Catholic Biblical Quarterly, 45 , p. 608-616.

Cavallin, H.C.C. (1974), Life After Death: Paul's Argument for the Resurrection of the Dead in I Cor 15. 1. Enquiry into the Jewish Background, Lund, CWK Gleerup (Coniectanea biblica. New Testament Series 7).

29. On interprète constamment $\mathrm{Rm}$ 7,7-25 en essayant obstinément d'identifier ce que le texte refuse d'identifier tout aussi obstinément: le référent qui se cache derrière l'énonciateur (ou le personnage ?) «je ». Le «je» anonyme mis en scène est-il Adam, Moïse, Paul, un Juif, un craignant-Dieu? Pourtant, il me semble qu'une approche purement textuelle (de type narratologique ou sémiotique) pourrait faire voir d'autres aspects du texte. 
Conzelmann, H. (1975) [allemand 1969], 1 Corinthians, Philadelphia, Fortress (Hermeneia).

Cooper, J.W. (2001) [1989], Body, Soul and Life Everlasting: Biblical Anthropology and the Monism-Dualism Debate, Grand Rapids - Cambridge - Leicester, Eerdmans - Apollos.

Cossette, R. et J. PePin (2001), «Vieillir et croître à travers les déclins, un défi spirituel avant tout», Théologiques, 9, p. 47-67.

Gignac, A. (2001), "Comprendre notre résurrection dans une perspective paulinienne. Les images de 1Th 4,13-18, 1Co 15 et 2Co 5,1-10", dans O. Mainville et D. Marguerat, dir., Résurrection. L'après-mort dans le monde ancien et le Nouveau Testament, Montréal - Genève, Médiaspaul Labor et Fides (Sciences bibliques 10 / Lire la Bible 45), p. 279-305.

- (2002), "Taubes, Badiou, Agamben: Reception of Paul by Non-Christian Philosophers Today", dans Society of Biblical Literature Seminar Papers 2002, Atlanta, Society of Biblical Literature, p. 74-110.

Glancy, J.A. (1998), "Obstacles to Slave's Participation in the Corinthian Church", Journal of Biblical Literature, 117, p. 481-501.

Green, J. (1999), "Restoring the Human Person: New Testament Voices for a Wholistic and Social Anthropology ", dans R.J. Russell, N. Murphy, T.C. Meyering et M.A. Arbib, dir., Neuroscience and the Person: Scientific Perspectives on Divine Action, Vatican City - Berkeley - Notre Dame, Vatican Observatory Publications - Center for Theology and Natural Sciences - University of Notre Dame Press, p. 3-22.

GuÉnel, V., dir. (1983), Le corps et le corps du Christ dans la première épître aux Corinthiens, Paris, Cerf (Lectio Divina 114).

Gundry, R.H. (1976), Sôma in Biblical Theology: With Emphasis on Pauline Anthropology, Cambridge, Cambridge University Press (SNTS Monograph Series 29).

Hays, R.B. (1997), First Corinthians, Louisville, John Knox Press (Interpretation).

Jewett, R. (1971), Paul's Anthropological Terms. A Study of their Use in Conflict Settings, Leiden, Brill.

Käsemann, E. (1982) [1964], Essays on New Testament Themes, Philadelphia, Fortress.

Martin, D.B. (1995), "The Resurrected Body", dans The Corinthian Body, New Haven - London, Yale University Press, p. 104-136. 
Morissette, R. (1972a), «L'antithèse entre le "psychique" et les "pneumatiques” en I Corinthiens XV, 44 à 46 ", Revue des sciences religieuses, 46, p. 97-143.

_ (1972 $)$, «L'expression "sôma" en 1 Cor 15 et dans la littérature paulinienne ", Revue des sciences philosophiques et théologiques, 56, p. 223-239.

- $\left(1972^{\mathrm{c}}\right)$, «La condition de ressuscité. 1 Corinthiens 15,35-49: structure littéraire de la péricope", Biblica, 53, p. 208-228.

Murphy, N. (1999), «Introduction», dans R.J. Russell, N. Murphy, T.C. Meyering et M.A. Arbib, dir., Neuroscience and the Person: Scientific Perspectives on Divine Action, Vatican City - Berkeley - Notre Dame, Vatican Observatory Publications - Center for Theology and Natural Sciences - University of Notre Dame Press, p. i-xxxv.

Murphy-O'Connor, J. (1978), "Corinthians Slogans in 1 Cor 6: 12-20", Catholic Biblical Quarterly, 40, p. 391-396.

Perrot, C. (2000), Après Jésus: le ministère chez les premiers chrétiens, Paris, L'Atelier (Vivre, croire, célébrer. Série Recherches).

Peters, T. (2002), "Introduction, What Is to Come», dans T. Peters, R.J. Russell et M. Welker, dir., Resurrection: Theological and Scientific Assessments, Grand Rapids, Eerdmans, p. viii-xvii.

Riccur, P. (1975), La métaphore vive, Paris, Seuil (L'ordre philosophique).

- (1986) [1970] "Qu'est-ce qu'un texte?", dans Du texte à l'action. Essais d’herméneutique II, Paris, Seuil, p. 137-159.

Robinson, H.W. (1913), The Christian Doctrine of Man, Edinburgh, T. \& T. Clark.

Robinson, J.A.T. (1952), The Body: A Study in Pauline Theology, London, SCM Press.

Rosner, B.S. (1994), Paul, Scripture and Ethics: A Study of 1 Corinthians 5-7, Leiden - New York, Brill (Arbeiten zur Geschichte des antiken Judentums und des Urchristentums 22).

Russell, R.J. (2002), «Bodily Resurrection, Eschatology, and Scientific Cosmology ", dans T. Peters, R.J. Russell et M. Welker, dir., Resurrection: Theological and Scientific Assessments, Grand Rapids, Eerdmans, p. 330 .

Sandnes, K.O. (2002), Belly and Body in the Pauline Epistles, Cambridge, Cambridge University Press (SNTS Monograph Series 120). 
Senft, C. (1990) [1979], La première épître de Saint-Paul aux Corinthiens, Genève, Labor et Fides (Commentaire du Nouveau Testament, $2^{\mathrm{e}}$ série, 7).

Son, S.-W. (2001) Corporate Elements in Pauline Anthropology: A Study of Selected Terms, Idioms, and Concepts in the Light of Paul's Usage and Background, Rome, Editrice Pontificio Istituto Biblico (Analecta biblica 148).

Theissen, G. (1996) [allemand 1975], "Les forts et les faibles à Corinthe. Analyse sociologique d'un conflit théologique », dans Histoire sociale $d u$ christianisme primitif: Jésus, Paul, Jean / trad. par I. Jaillet et A.-L. Fink, Genève, Labor et Fides (Monde de la Bible 33), p. 139-160 [= Evangelische Theologie, 35, p. 155-172].

Vergote, A. (1979), «Le Corps. Pensée contemporaine et catégories bibliques », Revue théologique de Louvain, 10, p. 159-175.

Vouga, F. (2000), «La nouvelle création et l'invention du moi », Études théologiques et religieuses, 75, p. 335-347.

Wiéner, C. (1983), "Notes sur 1 Corinthiens 6,12-20 », dans V. GuÉNEL, dir., Le corps et le corps du Christ dans la première épitre aux Corinthiens, Paris, Cerf (Lectio Divina 114), p. 88-93. 
Figure 2. - Hiérarchie épistémique et méthodes analogues. Un modèle des rapports science / théologie selon Russell $(2002,12)$.

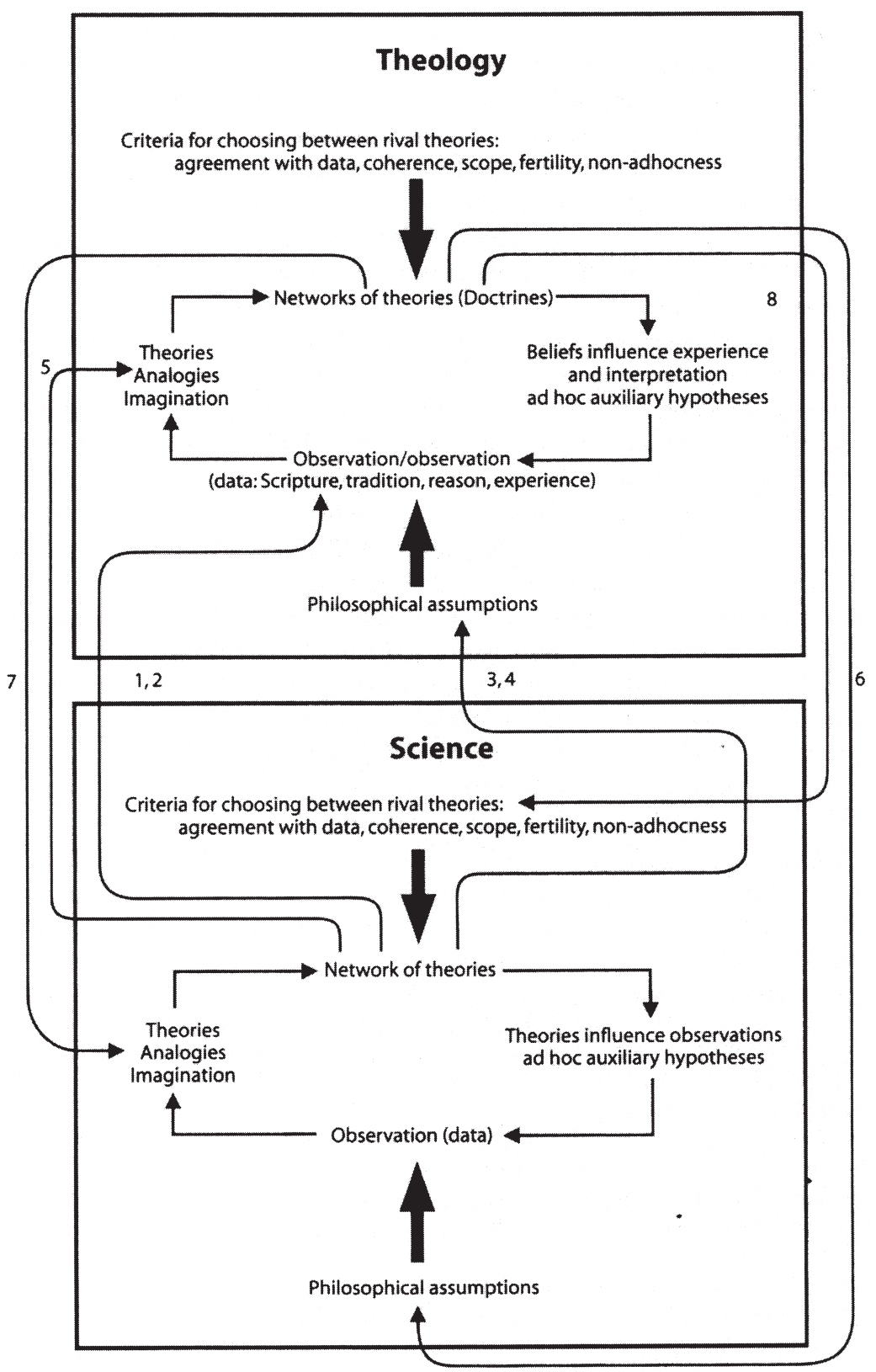




\title{
Résumé
}

S'adressant aux premières communautés chrétiennes des années 50 de l'ère commune, aux prises avec des problèmes concrets, Paul de Tarse crée sur le vif des réponses susceptibles de les guider. Il le fait à partir de ses intuitions théologiques, mais aussi de sa culture gréco-romaine et juive. Ce faisant, sans vouloir systématiser UNE anthropologie, il «jongle» avec quelques mots clés qui permettront à ses interprètes ultérieurs de construire une pluralité d'anthropologies chrétiennes (par exemple, Augustin, Thomas d'Aquin, Bultmann). En réexaminant l'utilisation des mots psychè, sarx, sôma et pneuma dans certains textes (1 Co 2,10-3,4; 1Co 6,12-20; 1Co 15,35-53) on arrive à deux constats. D'une part, chacun de ces mots désigne le Soi en entier, mais sous différents aspects: respectivement, l'action, la finitude (ou le péché), le choix et la relation à Dieu. D'autre part, l'anthropologie paulinienne, en autant qu'elle se laisse saisir, reflète une vision apocalyptique dont l'interprétation doit tenir compte. La croix et la résurrection de Jésus sont interprétées comme l'événement qui déclenche l'avènement d'un nouvel âge du monde, donc d'une humanité renouvelée et différente.

\begin{abstract}
Addressing concrete problems of the first century Christian communities, Paul of Tarsus, writing in the 50s C.E., formulates sensitive responses to guide them in the heat of the action. He answers from his unique theological mindset, but also in keeping with his Greco-Roman and Jewish culture. In doing this, but without proposing one systematized anthropology, he plays with several key words that permit later interpreters to build a variety of Christian anthropologies (for example, Augustine, Thomas Aquinas, Bultmann). In re-examining the use of the words, psyche, sarx, soma and pneuma in certain texts, such as, I Cor. 2:103:4; I Cor. 6:12-20; I Cor. 15:35-53, we arrive at two conclusions. On the one hand, each of these words indicates the whole self, but in different aspects, respectively: action, finiteness (or sin), choice and relationship to God. On the other hand, the Pauline anthropology, inasmuch as it can be grasped, reflects an apocalyptic vision whose interpretation must be taken into account. The cross and the resurrection of Jesus are interpreted as the event that triggers the advent of the new world order and a humanity that is renewed and different.
\end{abstract}

(C) Revue Théologiques 2004. Tout droit réservé. 